\title{
PRECIPITAÇÃO OBSERVADA NA AMAZÔNIA BRASILEIRA: COMPARAÇÃO ENTRE OS DADOS DAS REDES CONVENCIONAIS E DADOS DA REANÁLISE I DO NCEP/NCAR, CRU E GPCC ${ }^{1}$
}

\author{
LIMBERGER, Leila - leila.limberger@unioeste.br \\ Universidade Estadual do Oeste do Paraná / UNIOESTE \\ SILVA, Maria Elisa Siqueira - elisasiq@usp.br \\ Universidade de São Paulo / USP
}

\begin{abstract}
RESUMO: O avanço das tecnologias de informação relacionado à coleta de dados meteorológicos e ao seu compartilhamento através da internet permite o acesso a uma quantidade significativa de dados relativamente confiáveis para a maior parte do globo, a partir de um computador conectado à internet. Séries temporais incompletas e espacialmente mal distribuídas constituem um dos fatores que levam à reconstrução de dados de precipitação. Dados precisos de precipitação interpolados para grades espaciais regulares constituem uma variável importante para a caracterização climática. A totalidade de variáveis atmosféricas usadas comumente para a explicação da variabilidade da precipitação, obtidas através dos conjuntos de reanálises geradas por modelos numéricos em diversos centros mundiais, é fornecida em grades espacialmente regulares. O objetivo da presente pesquisa é avaliar, através da análise de estatísticas adequadas, a precisão dos dados de precipitação mensal para a bacia amazônica brasileira organizados pelos centros de pesquisa CRU, GPCC e os obtidos da Reanálise I do NCEP/NCAR em comparação com dados observados organizados pela ANA do Brasil, para o período de 1979 a 2010. Os dados interpolados para grades regulares usados neste estudo foram todos obtidos do ESRL/NOAA. Os valores dos coeficientes de correlação linear calculados, para cinco sub-regiões consideradas na bacia amazônica, entre a precipitação diretamente observada, da ANA, e aquela gerada pelos centros CRU, GPCC e NCEP/NCAR I são altos e significativos, o que demonstra que a utilização de dados reconstruídos e dispostos em grades regulares pode ser aplicada com alta confiabilidade, permitindo a expansão dos estudos em Climatologia Geográfica para as atuais necessidades de avanço dessa disciplina. Os resultados também mostram que a precipitação do conjunto da Reanálise I do NCEP/NCAR apresenta amplitude sazonal bastante inferior à observada nos demais conjuntos de dados usados. O conjunto de dados de grade que apresentou os maiores valores de correlação linear com a precipitação diretamente observada na bacia amazônica, da ANA, foi o GPCC.
\end{abstract}

PALAVRAS-CHAVES: dados de precipitação, Amazônia brasileira, CRU, GPCC, reanálise I do NCEP/NCAR.

OBSERVED PRECIPITATION IN THE BRAZILIAN AMAZON: COMPARISON BETWEEN DATA SET FROM THE CONVENTIONAL RAIN GAUGE NETWORKS AND REANALYSIS I DATA SET FROM THE NCEP/NCAR, CRU AND GPCC

ABSTRACT: The advancement of information technology related to the meteorological collection data and their sharing over the internet has allowed access to a significant amount of relatively reliable data by most part of the world, from a computer connected to the internet. Incomplete and poorly spatially distributed time series are one of the factors that lead to the reconstruction of precipitation data. Interpolation of accurate precipitation data onto regular spatial grids is an important variable for climate characterization. The totality of commonly used atmospheric variables to explain the variability of precipitation, obtained from the reanalysis data sets generated by numerical models in several worldwide centers, is provided on regular spatial grids. The purpose of

\footnotetext{
${ }^{1}$ Pesquisa realizada com recursos do $\mathrm{CNPq}$, através de concessão de Bolsa de Doutorado.
} 
this research is to evaluate, through appropriate statistical analysis, the accuracy of monthly precipitation data for the Brazilian Amazon basin organized by research centers CRU, GPCC and those derived from the NCEP/NCAR Reanalysis I compared to data observed and organized by ANA (National Water Agency) of Brazil for the period 19792010. The data interpolated onto regular grids in this study were all obtained from the ESRL/NOAA. The measured values of the linear correlation coefficient for the different considered subregions in the Amazon basin, between precipitation directly observed, by ANA, and that one generated by the centers CRU, GPCC and NCEP/NCAR I are high and significant, meaning that the use of reconstructed and arranged data on regular grids may be applied with high reliability, allowing the expansion of studies in Geographical Climatology for the increasing urgency to advance this discipline. The results also indicate that precipitation from the NCEP/NCAR Reanalysis I data sets presents significantly lower seasonal amplitude to that one observed in the other used data sets. The gridded data set that showed the highest values of linear correlation with the precipitation directly observed in the Amazon basin, by ANA, was the GPCC.

KEYWORDS: Precipitation dataset, Brazilian Amazon, CRU, GPCC, NCEP/NCAR Reanalysis I

\section{INTRODUÇÃO}

Ainda bastante recente no campo das ciências ambientais, a democratização de dados meteorológicos, por meio de processos simples de aquisição de dados (download) de grandes centros mundiais de pesquisa, traz novo fôlego às pesquisas em Climatologia (DEE et al., 2016). O acesso aos dados interpolados para grades regulares ajuda a resolver um dos principais problemas das pesquisas em Climatologia: a dificuldade de obtenção de dados. As séries de dados observados normalmente são curtas, especialmente na América do Sul, com muitas falhas e pouco densas espacialmente. Mesmo considerando-se as normas relativas à observação de dados meteorológicos (VAREJÃO-SILVA, 2006) sua confiabilidade deve ser avaliada quando se pretende realizar estudos científicos. São frequentes os relatos de problemas em estações meteorológicas, especialmente com pluviômetros, que podem tanto ser instalados em locais inadequados quanto apresentar erros nas coletas de dados, ou ainda, apresentar problemas técnicos diversos (LEIVAS et al., 2011). Além da irregularidade espacial e temporal dos dados diretamente observados, a caracterização climática de cada localidade e o estudo dos processos termodinâmicos que definem a precipitação é mais facilmente realizada a partir de dados padronizados (DEE et al., 2016).

Os dados interpolados para grades regulares podem se referir a conjuntos de reanálise, obtidos por sensoriamento remoto ou dados de previsão de tempo ou climática obtidos de modelagem numérica (FASULLO, 2012).

As reanálises constituem conjuntos de dados que podem ser usados para estudos climáticos e de tempo. São produzidas por grandes centros de pesquisa meteorológica, como os americanos NOAA (National Oceanic and Atmospheric Administration), NCAR (National Center for Atmospheric Research) e NASA (National Aeronautics and Space Administration), o europeu ECMWF (European Centre for Medium-Range Weather Forecast), o japonês JMA (Japan Meteorological Agency) (FASULLO, 2012). Os conjuntos de reanálises são constituídos por dados provenientes de modelagem numérica. Esses conjuntos constituem matrizes de dados referentes ao instante inicial usado em cada integração matemática realizada pelos modelos numéricos, com o objetivo de elaborar a previsão de tempo. Antes de ser informado ao modelo numérico, o 
conjunto de dados observados em superfície e altitude (obtidos principalmente através de estações meteorológicas convencionais, automáticas, sondagem vertical, boias em oceanos e sensoriamento remoto) é consistido com a aplicação de técnicas estatísticas e, interpolados para a grade do modelo usado. Após este procedimento de consistência e interpolação de dados, que faz parte da inicialização (HOLTON, 1992), o modelo numérico é executado de forma a gerar as previsões de tempo (DEE et al., 2016). A execução do modelo numérico para períodos pretéritos requer o uso de condições iniciais geradas anteriormente. Diversos centros meteorológicos ao redor do globo, tais como NCEP, NCAR, ECMWF e JMA (FASULLO, 2012) geram conjuntos de reanálise. Como cada centro tem, em geral, seu próprio modelo numérico operacional, os conjuntos de reanálise apresentam diferenças entre si, o que leva à realização de inúmeros estudos de comparação de conjuntos distintos de reanálises (TRENBERTH et al., 2005; TRENBERTH et al., 2011; FASULLO, 2012; HARADA et al., 2016). Como os modelos são executados para períodos passados relativamente extensos, pode-se gerar conjuntos de dados que permitam a realização de análises climáticas diagnósticas com base em dados regularmente espaçados. Um conjunto de dados de reanálise é gerado pela combinação entre dados observados e o esquema de assimilação de cada modelo numérico de previsão de tempo. Com estes procedimentos, em cada execução do modelo numérico cria-se uma matriz de dados regularmente espaçados que representa uma estimativa bastante precisa do estado atmosférico presente, para cada ponto de grade (DEE et al., 2016) e para cada nível atmosférico.

A reanálise atmosférica NCEP/NCAR I (KALNAY et al., 1996), elaborada nos anos 1990, é considerada a primeira geração desse tipo de produto. À essa reanálise não foram assimilados dados de vapor d'água do sensor especial imageador de micro-ondas (Special Sensor Microwave Imager - SSM/I) (TRENBERTH et al., 2005), assim como também não foram utilizados dados de vapor d'água de canais de infravermelho de outros satélites, nem de umidade sobre os oceanos (TRENBERTH et al., 2005 e 2011). Por esses motivos, este conjunto de reanálise apresenta, na maioria das vezes, problemas envolvendo a variabilidade espacial e temporal (especialmente a sazonalidade) dos dados, bem como valores muito baixos de transporte de umidade (TRENBERTH et al., 2005 e 2011).

Zveryaev e Chu (2003) analisaram dados de chuva do NCEP/NCAR I para os períodos de 1979 a 1998 e de 1948 a 1998 e identificaram que o padrão de variabilidade dominante tem características espúrias (não confiáveis) quanto à tendência.

Trenberth e Guillemot (1998) analisaram a reanálise I do NCEP/NCAR e identificaram problemas importantes, tais como "olhos de peixe" em estações em locais isolados, indicando uma grande influência do modelo nestes erros e problemas com os dados iniciais e parametrizações do modelo numérico. Visando incrementar a reanálise I do NCEP/NCAR, foi desenvolvido o projeto NCEP/NCAR II (ou NCEP-DOE), com dados desde 1979, que resolve alguns problemas, mas continua apresentando uma série de outros, ligados principalmente ao vapor d'água (TRENBERTH et al., 2005).

A segunda geração de reanálises, desenvolvidas já nos anos 2000, tem dois principais produtos: o ERA-40 (ECMWF 40-years Reanalysis) e o JRA-25 (Japanese 25-years Reanalysis). Apesar de terem apresentado melhorias, 
problemas quanto ao vapor d'água persistem nessas reanálises mais recentes (FASULLO, 2012).

A partir de 2010 alguns projetos de reanálise (que podem ser chamados de terceira geração) apresentaram melhorias quanto à resolução, inicialização e técnicas de assimilação dos dados, além de incorporarem faixas espectrais adicionais referentes às informações provenientes de satélites meteorológicos e de aperfeiçoarem os modelos. Dentre as reanálises da assim chamada terceira geração despontam a CFSRR (NCEP Coupled Forecast System Reanalyses and Reforecast) (SAHA et al., 2010), o MERRA (NASA's Modern Era Retrospective Analysis for Research and Applications) (RIENECKER et al., 2011), o Era-Interim (do ECMWF) (SIMMONS et al., 2007) e a JRA-55 (HARADA et al., 2016)

Admite-se que todos os dados de reanálise não conseguem ainda superar as limitações relativas à umidade (FASULLO, 2012; TRENBERTH et al., 2011), o que dificulta estudos de aspectos hidrológicos, especialmente quanto à tendência e variabilidade. Conjuntos de dados específicos são gerados no âmbito de projetos de intercomparação de reanálises, tal como o CMIP3 (Coupled Model Intercomparison Project phase 3) (MEEHL et al., 2007) e são frequentemente usados em pesquisas científicas: GFS Model Output (Global Forecast Service) e GHCN-3 (Gridded Land Precipitation and Temperature Anomalies).

Como a precipitação é uma variável difícil de ser simulada pelos modelos numéricos, uma vez que depende do bom desempenho do modelo tanto na descrição dos processos dinâmicos como termodinâmicos da atmosfera, existem várias outras tentativas de se gerar conjuntos globais equidistados a partir de dados observados em estações de superfície e por sensoriamento remoto (YIN et al., 2004; FASULLO, 2012). Os principais exemplos de dados de precipitação obtidos a partir de observação em superfície e sensoriamento remoto são o CHIRPS (Climate Hazards Group InfraRed Precipitation) (FUNK et al., 2015), o GPCP (Global Precipitation Climatology Project) (ADLER et al., 2001), o GPCC (Global Precipitation Climatology Centre) (SCHNEIDER et al., 2011), o CRU (Climate Research Unit) (BROHAN et al., 2006) e o TRMM (Tropical Rainfall Measuring Mission) (HUFFMAN et al., 2007).

Qualquer um desses conjuntos de dados pode ser utilizado para uma grande quantidade de estudos que buscam analisar a variabilidade temporal ou espacial de variados fenômenos atmosféricos, tendências, médias etc. Gulizia e Camiloni (2015) analisaram o comportamento mensal da chuva na América do Sul segundo três conjuntos de dados (CRU, GPCC e o da Universidade de Delaware - UDel) e verificaram que, de forma geral, há pequenas variações entre eles, não estatisticamente significativas.

Além dos conjuntos de dados mencionados aqui, há muitos outros usados pela comunidade científica, além de fontes de dados regionais, estaduais e nacionais. As vantagens mais importantes apresentadas pela utilização de dados globais interpolados em grade espacialmente regular podem ser elencadas como: a possibilidade de espacialização dos dados de forma relativamente simples, inclusive em nível global e, portanto, a análise de padrões espaciais característicos; a longevidade e ausência de falhas nas séries temporais; o grande número de variáveis atmosféricas disponíveis (no caso dos conjuntos de reanálise); a consistência temporal e espacial dos dados; a resolução temporal, de horária a anual (DEE et al., 2016). Outra vantagem em se utilizar dados 
padronizados é a facilidade de comparação com resultados de outras fontes, permitindo a realização de estudos de confiabilidade entre diferentes fontes.

Procurando avaliar a qualidade dos conjuntos de precipitação mensal reconstruída, CRU, GPCC e Reanálise I do NCEP/NCAR, a presente pesquisa propõe identificar o comportamento espaço-temporal da chuva em cinco subregiões homogêneas da bacia amazônica brasileira, definidas em Limberger (2015), em comparação com dados diretamente observados em estações convencionais e automáticas de superfície, catalogados e disponibilizados pela Agência Nacional de Águas (ANA), através do seu sistema Hidroweb (http://hidroweb.ana.gov.br).

\section{MATERIAL E PROCEDIMENTOS METODOLÓGICOS}

Os dados mensais de precipitação a serem comparados fazem parte dos conjuntos organizados pela ANA (denominados de dados observados), CRU, GPCC e Reanálise I do NCEP/NCAR (denominados aqui de dados reconstruídos CRU, GPCC e NCEP/NCAR I), para o período de 1979 a 2010.

O conjunto de dados NCEP/NCAR I (KALNAY et al., 1996) estão dispostos em grade regular com espaçamento em longitude e latitude de 2,5 graus. Este conjunto de dados apresenta mais de duas dezenas de variáveis, em 17 níveis barométricos, com resolução temporal de seis horas, de 1948 até o presente. Os dados estão também disponíveis para as resoluções diária e mensal, em arquivos diferentes. Podem ser acessados pelo site <http://www.esrl.noaa.gov/psd/data/gridded/data.ncep.reanalysis.html>.

Pesquisadores do Global Precipitation Climatology Centre (GPCC) reconstruíram dados observados de precipitação mensal global para uma grade espacialmente regular, com resolução espacial de 0,5 grau, de 1910 a 2010. A reconstrução foi realizada com base em 13 métodos estatísticos diferentes, que podem ser verificados no site <http://www.esrl.noaa.gov/psd/data/gridded/data.gpcc.html> (SCHNEIDER et al., 2011).

O Climate Research Unit (CRU), ligado ao instituto British Atmospheric Data Centre (BADC), constitui conjunto de dados obtidos a partir de observações, bastante usado pela comunidade científica. É composto por dados de temperatura do ar em superfície (mínima e máxima) e precipitação, com resolução espacial de 0,5 grau, tanto em latitude quanto em longitude, de 1910 a 2010. A máxima resolução temporal deste conjunto de dados é mensal. Pode tanto ser obtido pelo site do ESRL/NOAA como pelo https://badc.nerc.ac.uk/data, após cadastro (BROHAN et al., 2006).

Como os dados de precipitação da ANA não cobrem homogeneamente o espaço, tal como ilustrado pela localização dos postos de observação na Figura 1 , não podem ser diretamente comparados com os dados obtidos das demais fontes de dados, CRU, GPCC e Reanálise I NCEP/NCAR, distribuídos espacialmente de forma regular. Assim, optou-se por comparar as séries temporais dos valores médios representativos de áreas espacialmente homogêneas da região amazônica. 


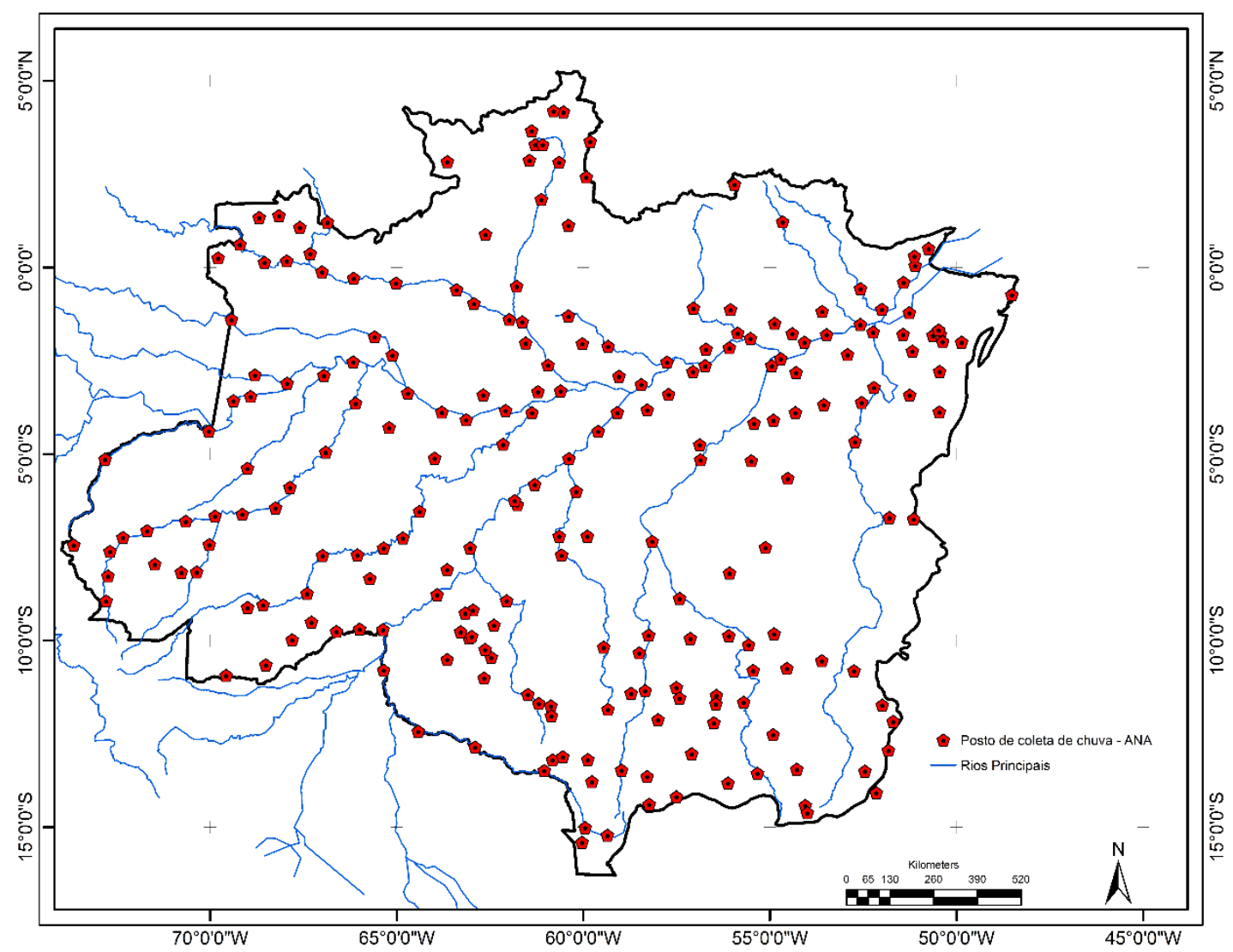

Figura 1 - Localização dos postos de coleta de precipitação da bacia amazônica brasileira disponibilizados pela ANA. São apresentados os rios mais expressivos da região.

Devido à sua grande dimensão, a bacia amazônica brasileira não apresenta comportamento climático semelhante em toda sua extensão. Desta forma, a identificação de áreas homogêneas quanto à precipitação permite que seja evidenciada a variabilidade espacial presente em toda a bacia. Tal como definido por Limberger (2015), as áreas homogêneas na bacia amazônica foram identificadas pela aplicação de análise multivariada de agrupamento aos dados de vazão fluvial mensal. A aplicação da mesma análise de agrupamento a dados de precipitação mensal forneceu resultados menos precisos para a definição das áreas homogêneas, tal como discutido em Limberger (2015). As sub-regiões homogêneas são denominadas de Norte, Amazonas-Foz, Oeste, Sul e Central e estão apresentadas na Figura 2. Nesta mesma figura são apresentados, para cada uma das sub-regiões consideradas, o ciclo anual médio da precipitação calculado para o período de 1978 a 2010, com dados da ANA. 


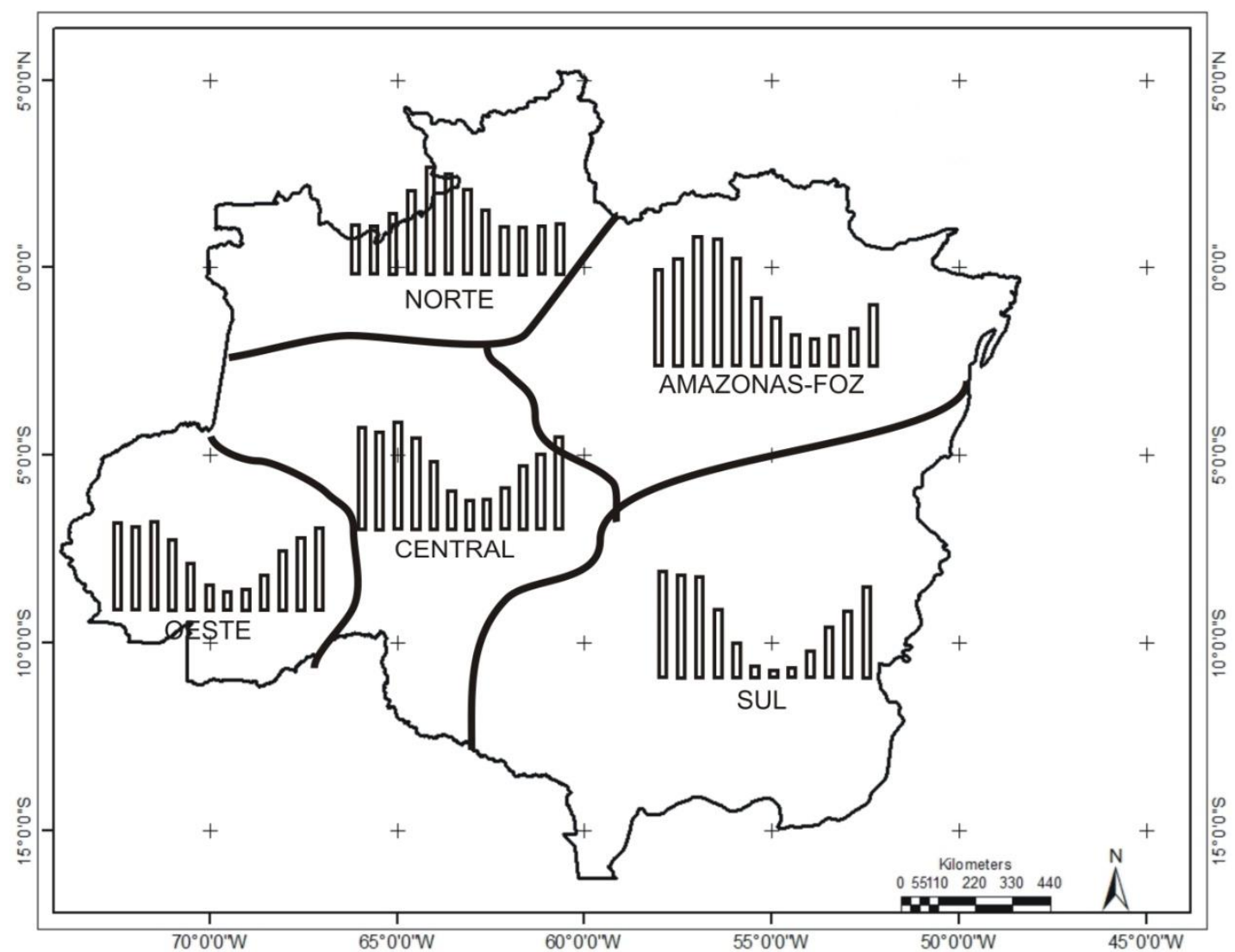

Figura 2 - Identificação das cinco sub-regiões homogêneas utilizadas na presente pesquisa: Norte, Amazonas-Foz, Oeste, Central e Sul, bem como o respectivo ciclo sazonal médio da precipitação observada, utilizando os dados da ANA. Os histogramas apresentam a mesma escala para o valor da precipitação mensal $(\mathrm{mm})$.

Fonte: Limberger (2015).

Os valores médios das séries temporais originais foram gerados para cada uma das cinco áreas homogêneas pré-estabelecidas, tanto para os dados observados diretamente, obtidos da ANA, como para os dados reconstruídos (CRU, GPCC e Reanálise I NCEP/NCAR). No caso dos dados da ANA, a média espacial foi calculada considerando-se todos os postos pluviométricos pertencentes a cada uma das sub-regiões homogêneas. Para os dados reconstruídos, a média da precipitação foi calculada para pontos internos às áreas retangulares aproximadamente coincidentes com as áreas definidas pelas sub-regiões da Figura 2, limitadas pelas coordenadas geográficas $(6900 ; 30 \mathrm{~N})$ e $\left(61^{\circ} \mathrm{O}, 1,5^{\circ} \mathrm{S}\right)$, sub-região Norte; $(6100 ; 00)$ e $(51,50 \mathrm{0}, 50 \mathrm{~S})$, sub-região Amazonas-Foz; (73o 0, $50 \mathrm{~S})$ e $(61,50 \mathrm{0} ; 10 \mathrm{o} \mathrm{S})$, Oeste; (60o 0; $50 \mathrm{~S})$ e (510 $0 ; 13,50 \mathrm{~S})$, Sul; 6600, $100 \mathrm{~S}$ e $6000,2,50 \mathrm{~S}$, Central, com o auxílio do software GrADS (Gridded Analysis and Display System). Nesta análise, não foram removidas a sazonalidade nem a tendência linear das séries temporais consideradas, tanto dos dados observados quanto dos reconstruídos.

Considerando-se que os dados do GPCC são registrados em $\mathrm{mm}$ dia-1 e os do NCEP/NCAR em $\mathrm{kg} \mathrm{m-2,} \mathrm{os} \mathrm{mesmos} \mathrm{foram} \mathrm{multiplicados} \mathrm{por} 30$ e 4, 
respectivamente, para que pudessem ser comparados com os demais, com total mensal registrado em milímetros.

A comparação entre os dados relativos aos diversos conjuntos considerados foi feita através do cálculo do coeficiente de correlação linear ( $r$. Para os cálculos de correlação linear e a elaboração de gráficos de dispersão (não apresentados neste artigo) foi utilizada a planilha eletrônica Microsoft Excel. A significância estatística dos resultados de correlação linear foi definida pelo teste t-Student.

Com intuito de se identificar a capacidade de cada conjunto de dados em representar os eventos extremos de chuva foram calculados o comportamento médio de cada um e o quanto variam entre si, valores médios, máximos e mínimos para cada conjunto de dados, além do desvio padrão.

\section{RESULTADOS E DISCUSSÃO}

Dentre todos os resultados do cálculo do coeficiente de correlação linear entre dados reconstruídos (NCEP/NCAR I, GPCC e CRU) e dados observados da ANA, apresentados na Tabela 1, os valores para o conjunto de dados do GPCC foram sempre os maiores, podendo-se destacar o coeficiente de correlação linear para a sub-região Sul, igual a 0,98. Todos os valores de correlação linear entre dados observados da ANA e os do GPCC são maiores que 0,95, o que é significativo estatisticamente para o nível de $99,5 \%$ de confiabilidade, de acordo com o teste t-Student. Os menores valores de correlação linear em comparação com os dados observados foram verificados para os dados da Reanálise I do NCEP/NCAR, porém, ainda com significância estatística superior a 99\%, destacando-se o valor máximo de 0,89 para a precipitação observada na subregião Sul. O menor valor de $r$ para os dados da Reanálise I NCEP/NCAR é igual a 0,64 , e ocorre para a precipitação observada na sub-região Norte. Dados da Reanálise I do NCEP/NCAR apresentam os menores valores de correlação dentre todos os resultados, como é conhecido na literatura (TRENBERTH e GUILLEMOT, 1998; LOS, 2015). Ainda, para a sub-região Norte, verifica-se que o maior valor de correlação linear entre os dados observados e reconstruídos ocorre para os dados do GPCC, com r igual 0,95 (Tabela 1).

Tabela 1 - Coeficiente de correlação linear entre a precipitação total mensal observada da ANA e a precipitação mensal reconstruída (CRU, Reanálise I do NCEP/NCAR e GPCC), para a série temporal de janeiro de 1979 a dezembro de 2010, para as sub-regiões Norte, Amazonas-Foz, Oeste, Sul e Central da bacia amazônica brasileira. Todos os valores apresentam significância estatística superior a 99\% (teste t-Student).

\begin{tabular}{lrrrrr}
\hline & Norte & AmazFoz & Oeste & Sul & Central \\
\hline ANA X GPCC & 0,95 & 0,98 & 0,96 & 0,98 & 0,96 \\
ANA X CRU & 0,87 & 0,95 & 0,86 & 0,96 & 0,92 \\
ANA X NCEP/NCAR I & 0,64 & 0,82 & 0,85 & 0,89 & 0,85 \\
\hline
\end{tabular}

A comparação da precipitação observada na sub-região Amazonas-Foz com os dados reconstruídos mostra valores altos de correlação linear, entre 0,82 e 0,98, em particular, para os conjuntos de dados da Reanálise I e GPCC 
(Tabela 1). Este resultado permite sugerir que os dados de chuva da sub-região Amazonas-Foz das quatro fontes de dados consideradas apresentam variabilidade temporal bastante similar entre si, o que não é o mesmo dizer que seus valores absolutos são parecidos, em especial no caso dos dados da Reanálise I do NCEP/NCAR, como abordado adiante.

Para o caso da sub-região Oeste, os resultados de correlação linear entre a série observada e as séries dos demais conjuntos de dados reconstruídos apresentam valores entre 0,85 e 0,96, como o maior valor de correlação para os dados do GPCC, e o menor valor para os dados da Reanálise I do NCEP/NCAR (Tabela 1).

A sub-região Sul apresenta os maiores valores de correlação linear. O menor ( $r=0,89$, entre NCEP/NCAR e dados observados) e o maior valor de correlação ( $r=0,98$, entre GPCC e observados) são bastante próximos de 1 , 0 que indica que os conjuntos de dados apresentam variabilidade mais semelhante. Assim, para essa sub-região, sugere-se que qualquer um dos conjuntos de dados reconstruídos possa ser utilizado sem prejuízo para as conclusões das pesquisas às quais for empregado, considerando-se, entretanto, que os dados da Reanálise I do NCEP/NCAR só podem ser considerados na forma de anomalia em relação à média, uma vez que os valores absolutos da precipitação associada ao conjunto do NCEP/NCAR são bastante distintos dos apresentados nos demais conjuntos de dados, como mostrado adiante.

Novamente, o menor valor de correlação linear para a precipitação da sub-região Central foi verificado entre os dados observados da ANA e os da Reanálise I do NCEP/NCAR $(r=0,84)$ e o maior, entre os dados da ANA e os do GPCC $(r=0,93)$.

A análise da correlação linear entre os conjuntos de dados reconstruídos mostra que dados do CRU e GPCC apresentam comportamentos mais semelhantes entre si no decorrer do tempo, enquanto que os dados do NCEP/NCAR apresentam correlações menores, tanto em relação aos conjuntos de dados reconstruídos quanto em relação aos dados observados da ANA. As diferenças entre os conjuntos de dados reconstruídos e os dados observados da ANA decorrem da aplicação de diferentes métodos estatísticos na compilação dos dados e dos diferentes conjuntos de dados primários compilados, tal como discutem Carvalho et al. (2012), e das médias espaciais aplicadas.

A comparação dos valores médios, máximos, mínimos e de desvio padrão da precipitação mensal para as cinco sub-regiões em estudo, para as quatro fontes de dados consideradas, conforme Tabela 2, permite verificar que os dados do CRU e do GPCC apresentam os valores mais semelhantes entre si, e muito próximo dos valores observados da ANA. Os valores da Reanálise I do NCEP/NCAR são os mais destoantes. Esse resultado corrobora os resultados já obtidos de correlação linear, indicando que o conjunto de dados que melhor representa os dados observados da ANA é o GPCC, para as sub-regiões Norte, Amazonas-Foz e Oeste; no caso das sub-regiões Sul e Central identifica-se ora maior semelhança com os dados do CRU, ora com os dados do GPCC.

Apesar do valor médio absoluto da precipitação da Reanálise I do NCEP/NCAR ser sempre menor do que o fornecido pela ANA, apresentando diferenças percentuais entre $-23,8 \%$ e $-31,2 \%$, de acordo com a sub-região, os valores de desvio padrão, ou variabilidade temporal, apresentam as maiores 
diferenças, entre $-79,5 \%$ e $-88,4 \%$. Porém, os valores da Reanálise I do NCEP/NCAR que mais destoam dos dados da ANA são os valores mínimos da precipitação no decorrer do ano, com diferenças que oscilam entre $119,7 \%$ e $2513,2 \%$, sempre maiores do que os observados.

Tabela 2 - Valor médio, máximo absoluto, mínimo absoluto e desvio padrão (em valor absoluto e percentual (em itálico) da precipitação mensal relativa aos conjuntos de dados do GPCC, CRU, NCEP/NCAR I e ANA, entre 1979 e 2010, para as sub-regiões Norte, Amazonas-Foz, Oeste, Sul e Central. As células em vermelho (azul) apresentam os valores com oscilação positiva (negativa) quanto aos valores de precipitação registrados pela ANA.

\begin{tabular}{|c|c|c|c|c|c|}
\hline & & GPCC & CRU & NCEP/NCAR I & ANA \\
\hline \multirow[t]{4}{*}{ Norte } & Média & $\begin{array}{r}225,92 \\
+12,5 \% \\
\end{array}$ & $\begin{array}{r}232,08 \\
+15,6 \% \\
\end{array}$ & $\begin{array}{c}138,14 \\
-31,2 \% \\
\end{array}$ & 200,73 \\
\hline & Máximo & $\begin{array}{l}445,94 \\
+2,5 \%\end{array}$ & $\begin{array}{r}526,98 \\
+21,1 \%\end{array}$ & $\begin{array}{l}164,48 \\
-62,2 \%\end{array}$ & 435,12 \\
\hline & Mínimo & $\begin{array}{r}52,85 \\
+7,5 \% \\
\end{array}$ & $\begin{array}{c}29,31 \\
-40,4 \% \\
\end{array}$ & $\begin{array}{r}108,03 \\
+119,7 \% \\
\end{array}$ & 49,17 \\
\hline & $\begin{array}{l}\text { Desvio } \\
\text { padrão }\end{array}$ & $\begin{array}{l}75,57 \\
-4,2 \% \\
\end{array}$ & $\begin{array}{r}87,47 \\
+10,9 \% \\
\end{array}$ & $\begin{array}{c}9,14 \\
-88,4 \% \\
\end{array}$ & 78,88 \\
\hline \multirow[t]{4}{*}{ Amazonas-Foz } & Média & $\begin{array}{l}186,23 \\
-0,7 \% \\
\end{array}$ & $\begin{array}{l}171,87 \\
-8,4 \% \\
\end{array}$ & $\begin{array}{r}142,84 \\
-23,8 \% \\
\end{array}$ & 187,53 \\
\hline & Máximo & $\begin{array}{l}416,40 \\
+0,4 \% \\
\end{array}$ & $\begin{array}{c}447,67 \\
+8 \% \\
\end{array}$ & $\begin{array}{l}175,37 \\
-57,7 \% \\
\end{array}$ & 414,60 \\
\hline & Mínimo & $\begin{array}{r}25,14 \\
+30,1 \% \\
\end{array}$ & $\begin{array}{r}15,25 \\
-21,1 \% \\
\end{array}$ & $\begin{array}{r}107,67 \\
+457,3 \% \\
\end{array}$ & 19,32 \\
\hline & $\begin{array}{l}\text { Desvio } \\
\text { padrão }\end{array}$ & $\begin{array}{l}103,91 \\
-2,3 \%\end{array}$ & $\begin{array}{r}99,13 \\
-6,8 \% \\
\end{array}$ & $\begin{array}{c}14,43 \\
-86,4 \% \\
\end{array}$ & 106,35 \\
\hline \multirow[t]{4}{*}{ Oeste } & Média & $\begin{array}{r}199,37 \\
+9,8 \% \\
\end{array}$ & $\begin{array}{l}188,33 \\
+3,7 \% \\
\end{array}$ & $\begin{array}{r}137,63 \\
-24,2 \% \\
\end{array}$ & 181,64 \\
\hline & Máximo & $\begin{array}{l}422,68 \\
+7,3 \% \\
\end{array}$ & $\begin{array}{r}514,88 \\
+30,7 \% \\
\end{array}$ & $\begin{array}{c}174,94 \\
-55,6 \% \\
\end{array}$ & 394,00 \\
\hline & Mínimo & $\begin{array}{c}16,22 \\
+355,6 \%\end{array}$ & $\begin{array}{c}11,32 \\
+218 \%\end{array}$ & $\begin{array}{c}93,03 \\
+2513,2 \%\end{array}$ & 3,56 \\
\hline & $\begin{array}{l}\text { Desvio } \\
\text { padrão }\end{array}$ & $\begin{array}{r}105,98 \\
+18,6 \% \\
\end{array}$ & $\begin{array}{r}108,55 \\
+21,5 \% \\
\end{array}$ & $\begin{array}{c}17,31 \\
-80,6 \% \\
\end{array}$ & 89,33 \\
\hline \multirow[t]{4}{*}{ Sul } & Média & $\begin{array}{r}173,24 \\
+4,88 \% \\
\end{array}$ & $\begin{array}{c}176,75 \\
+7 \%\end{array}$ & $\begin{array}{l}125,03 \\
-24,3 \% \\
\end{array}$ & 165,17 \\
\hline & Máximo & $\begin{array}{r}475,80 \\
+13,6 \% \\
\end{array}$ & $\begin{array}{l}393,10 \\
-6,2 \% \\
\end{array}$ & $\begin{array}{c}163,39 \\
-61 \% \\
\end{array}$ & 418,88 \\
\hline & Mínimo & $\begin{array}{c}2,42 \\
-41,3 \% \\
\end{array}$ & $\begin{array}{r}7,51 \\
+82,3 \% \\
\end{array}$ & $\begin{array}{r}75,96 \\
+1743,7 \% \\
\end{array}$ & 4,12 \\
\hline & $\begin{array}{l}\text { Desvio } \\
\text { padrão }\end{array}$ & $\begin{array}{l}123,61 \\
+9,1 \% \\
\end{array}$ & $\begin{array}{l}119,15 \\
+5,2 \% \\
\end{array}$ & $\begin{array}{c}20,12 \\
-82,2 \% \\
\end{array}$ & 113,27 \\
\hline \multirow[t]{4}{*}{ Central } & Média & $\begin{array}{r}208,10 \\
+11,8 \% \\
\end{array}$ & $\begin{array}{l}186,62 \\
+0,3 \% \\
\end{array}$ & $\begin{array}{r}141,69 \\
-23,8 \% \\
\end{array}$ & 186,06 \\
\hline & Máximo & $\begin{array}{r}430,46 \\
+10,7 \% \\
\end{array}$ & $\begin{array}{r}466,07 \\
+19,8 \% \\
\end{array}$ & $\begin{array}{l}174,47 \\
-55,1 \% \\
\end{array}$ & 388,97 \\
\hline & Mínimo & $\begin{array}{l}30,23 \\
-6,8 \% \\
\end{array}$ & $\begin{array}{r}24,96 \\
-23,1 \% \\
\end{array}$ & $\begin{array}{r}99,49 \\
+206,6 \% \\
\end{array}$ & 32,45 \\
\hline & $\begin{array}{l}\text { Desvio } \\
\text { padrão }\end{array}$ & $\begin{array}{c}105,61 \\
+28,8 \%\end{array}$ & $\begin{array}{c}100,79 \\
+22,9 \%\end{array}$ & $\begin{array}{c}16,83 \\
-79,5 \%\end{array}$ & 82,02 \\
\hline
\end{tabular}


A precipitação do GPCC apresenta valores médios, mínimos e máximos maiores do que os da ANA, para todas as sub-regiões, com exceção do valor médio na sub-região Amazonas-Foz e mínimos nas sub-regiões Sul e Central, que são inferiores aos valores da ANA. Apesar de os dados de precipitação mensal do CRU apresentarem comportamento bastante semelhante aos dados da ANA, bem como os dados do GPCC, os valores médios são maiores do que os registrados pela ANA, exceto para a sub-região Amazonas-Foz (-8,4\%). Quanto aos valores máximos de precipitação, somente as regiões Sul e Central apresentam valores menores do que os registrados pela ANA, com diferenças iguais a $-6,2$ e $-23,1 \%$, respectivamente. Já para os valores mínimos registrados pelo $\mathrm{CRU}$, as sub-regiões Oeste e Sul apresentam valores maiores do que os registrados pela ANA (em $218,0 \%$ e $82,3 \%$, respectivamente) e as demais, valores inferiores.

Considerando a análise da Tabela 2, portanto, conclui-se que os dados da Reanálise I do NCEP/NCAR são os menos adequados para o estudo de variabilidade sazonal e mensal. Corroborando essa afirmação, observa-se na Figura 3, onde estão representados os ciclos sazonais médios para os dados dos quatro conjuntos analisados e para as cinco sub-regiões consideradas, que a precipitação da Reanálise I do NCEP/NCAR (barra azul) apresenta sazonalidade mais fracamente definida em comparação aos dados das demais fontes. São apresentados valores muito altos para a estação seca, exceto para a sub-região Norte, e muito baixos para a estação chuvosa de cada sub-região. Por outro lado, verifica-se que a precipitação média do GPCC e CRU apresentam ciclos sazonais próximos entre si e em relação aos valores fornecidos pela ANA. A precipitação média mensal da Reanálise I do NCEP/NCAR nas sub-regiões Norte e Amazonas-Foz apresenta os menores valores de amplitude anual.

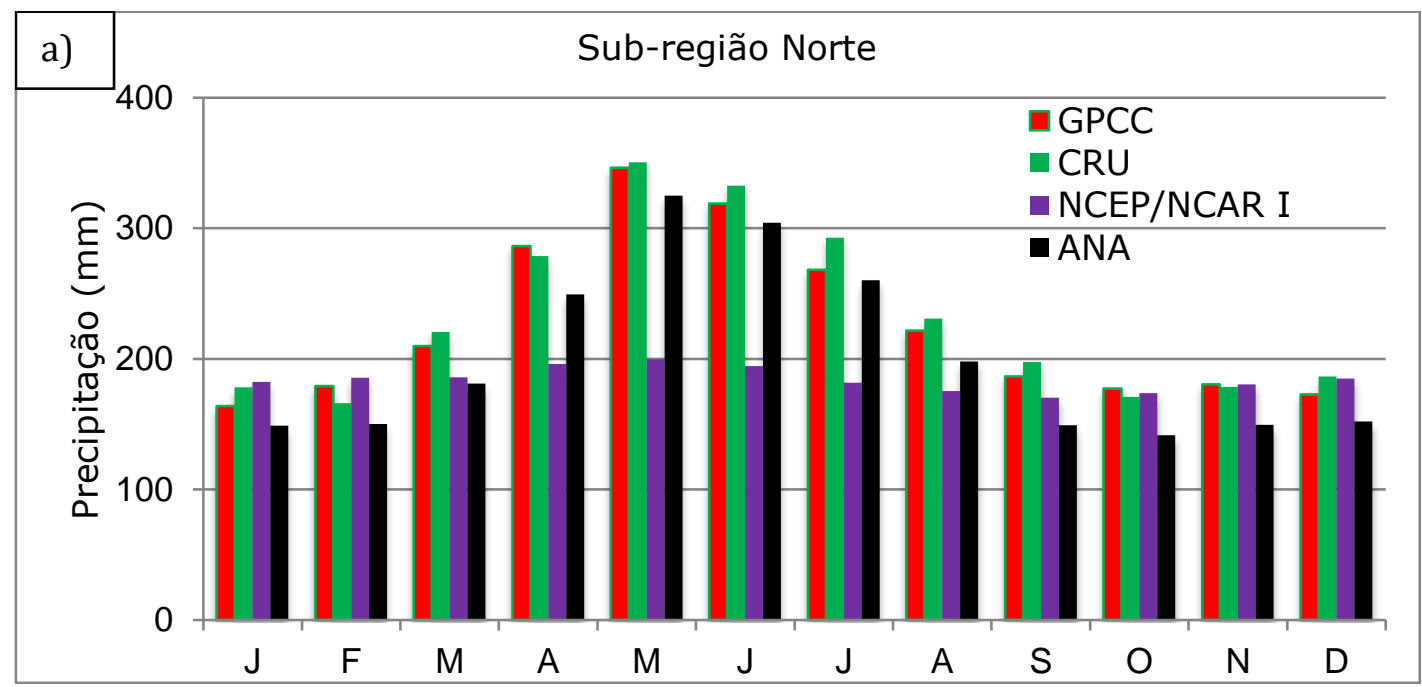




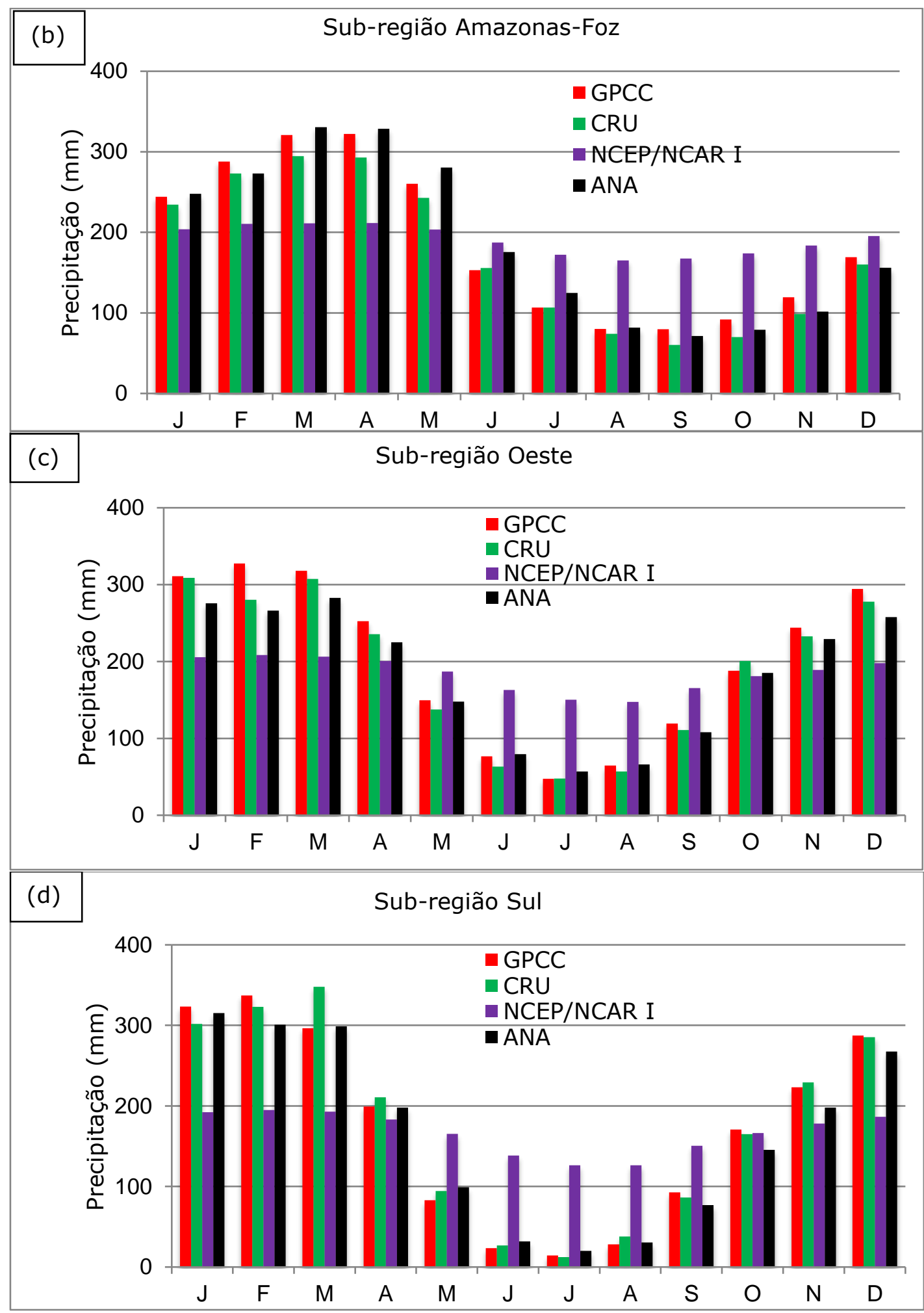


(e)

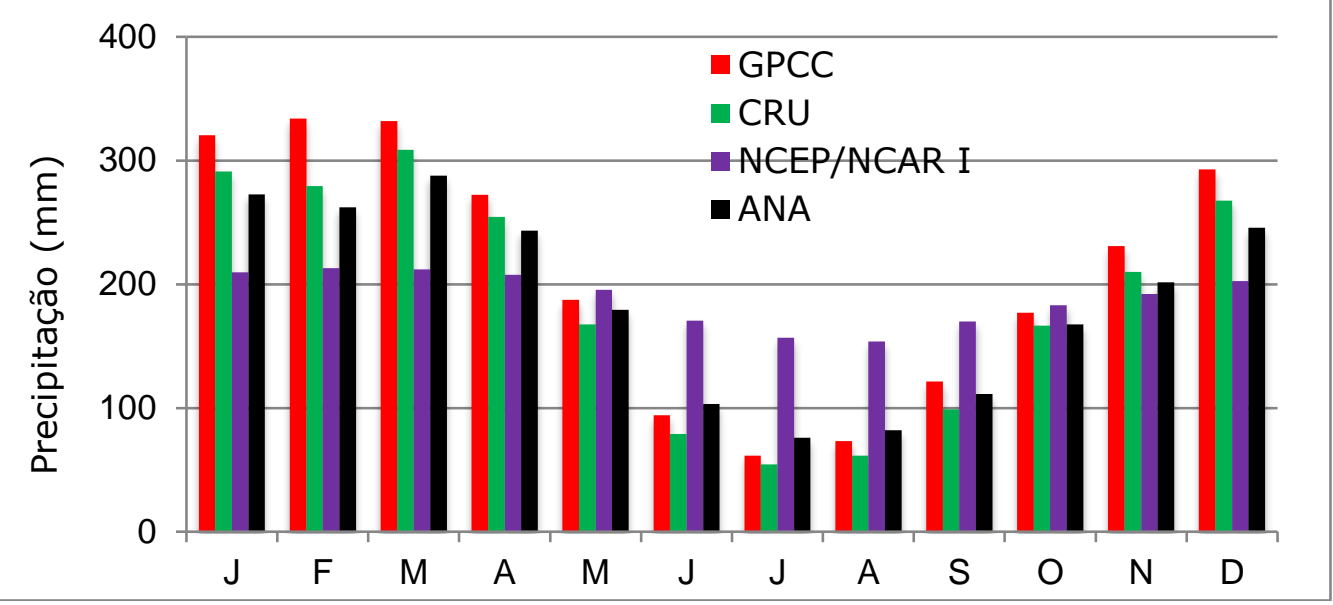

Figura 3 - Ciclo anual médio da precipitação para o período de 1979 a 2010, para os dados do GPCC (barra vermelha), CRU (barra verde), Reanálise I do NCEP/NCAR (barra roxa) e dados observados da ANA (barra preta), para as cinco sub-regiões homogêneas da região Norte do Brasil: (a) Norte, (b) Amazonas-Foz, (c) Oeste, (d) Sul e (e) Central.

As séries temporais dos dados mensais de precipitação para todos os conjuntos considerados, CRU, GPCC, Reanálise I do NCEP/NCAR e dados observados da ANA, evidenciam a diferença dos valores de precipitação da Reanálise I do NCEP-NCAR em relação às demais fontes, tanto do valor médio como da amplitude anual, para cada uma das sub-regiões consideradas, tal como visto na Figura 4.

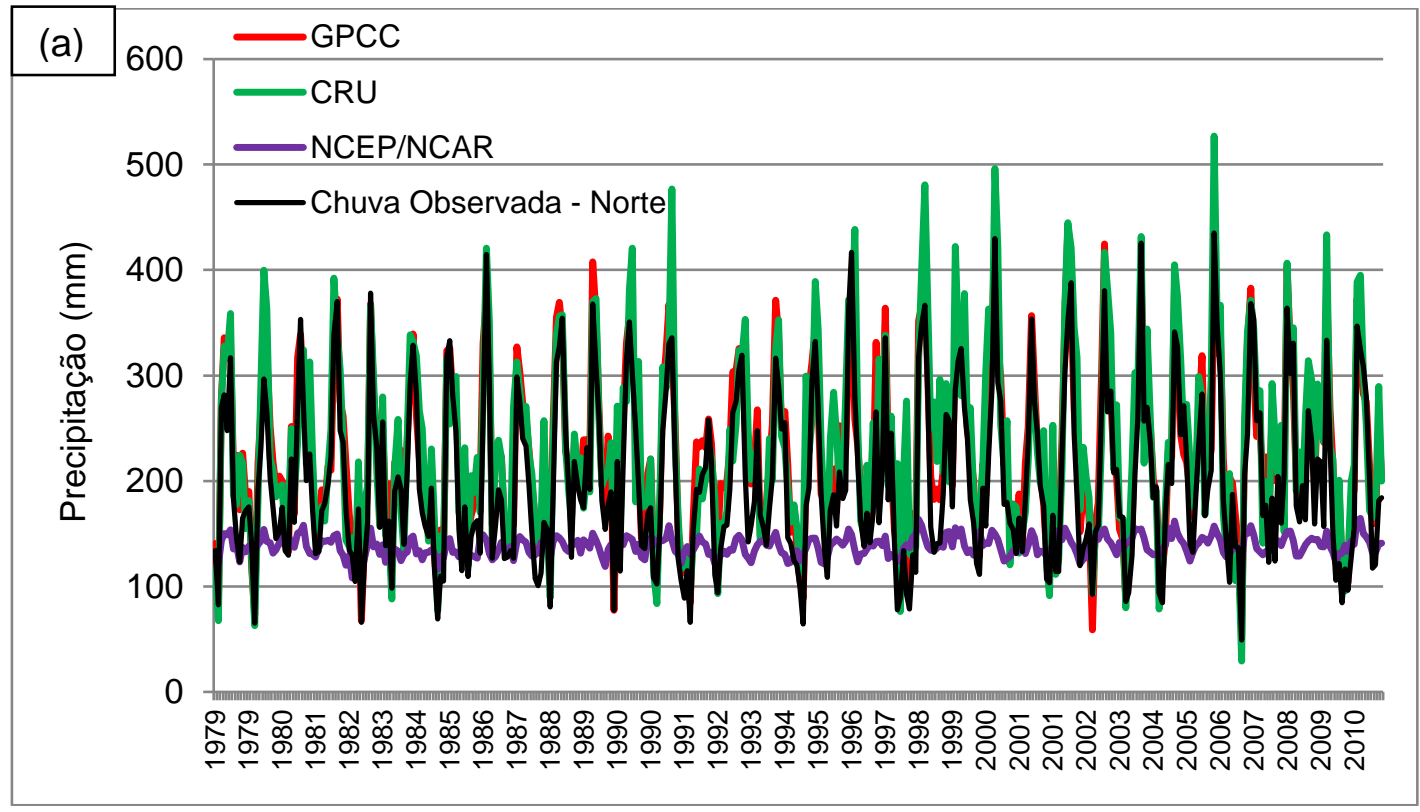




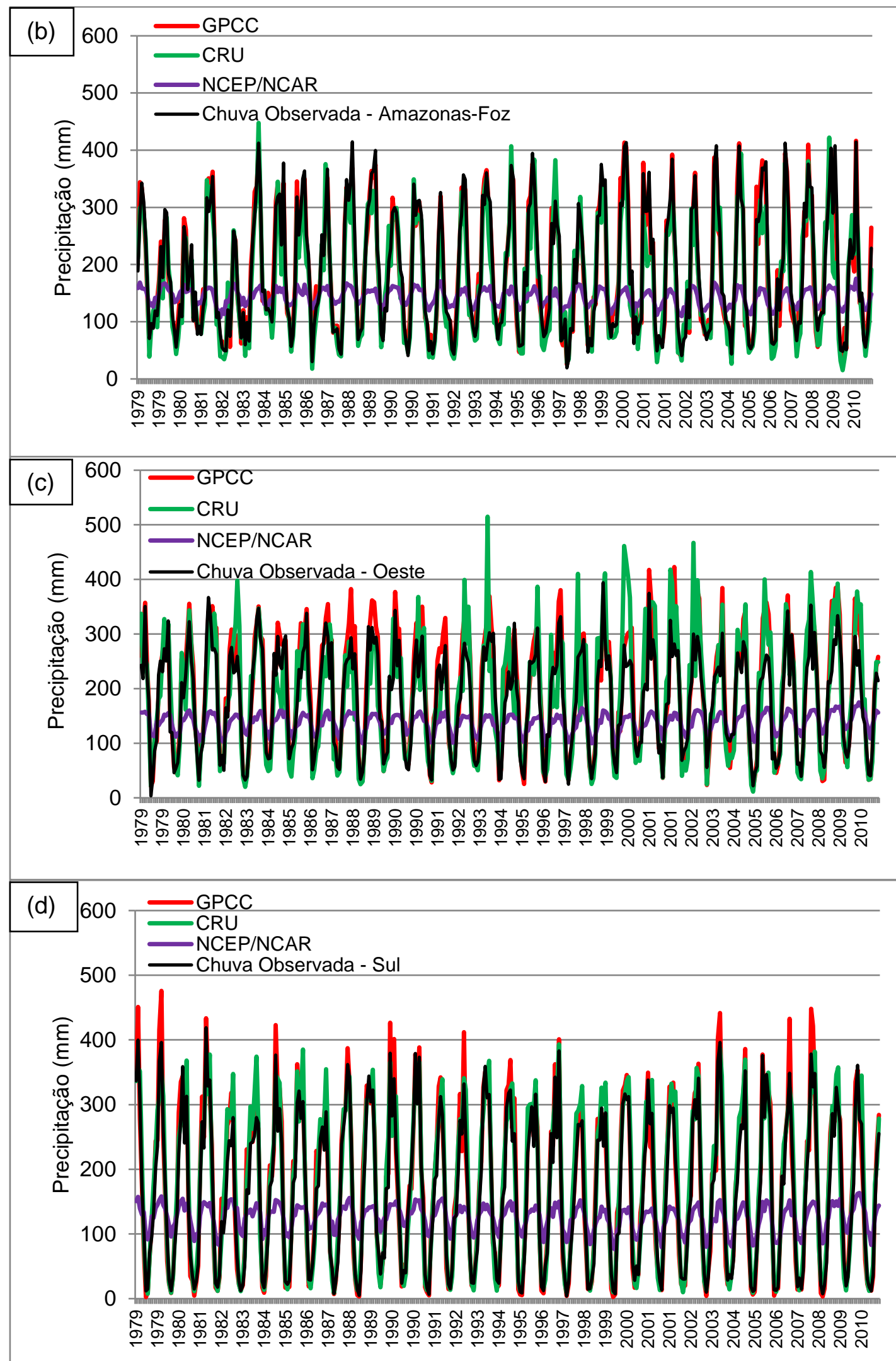




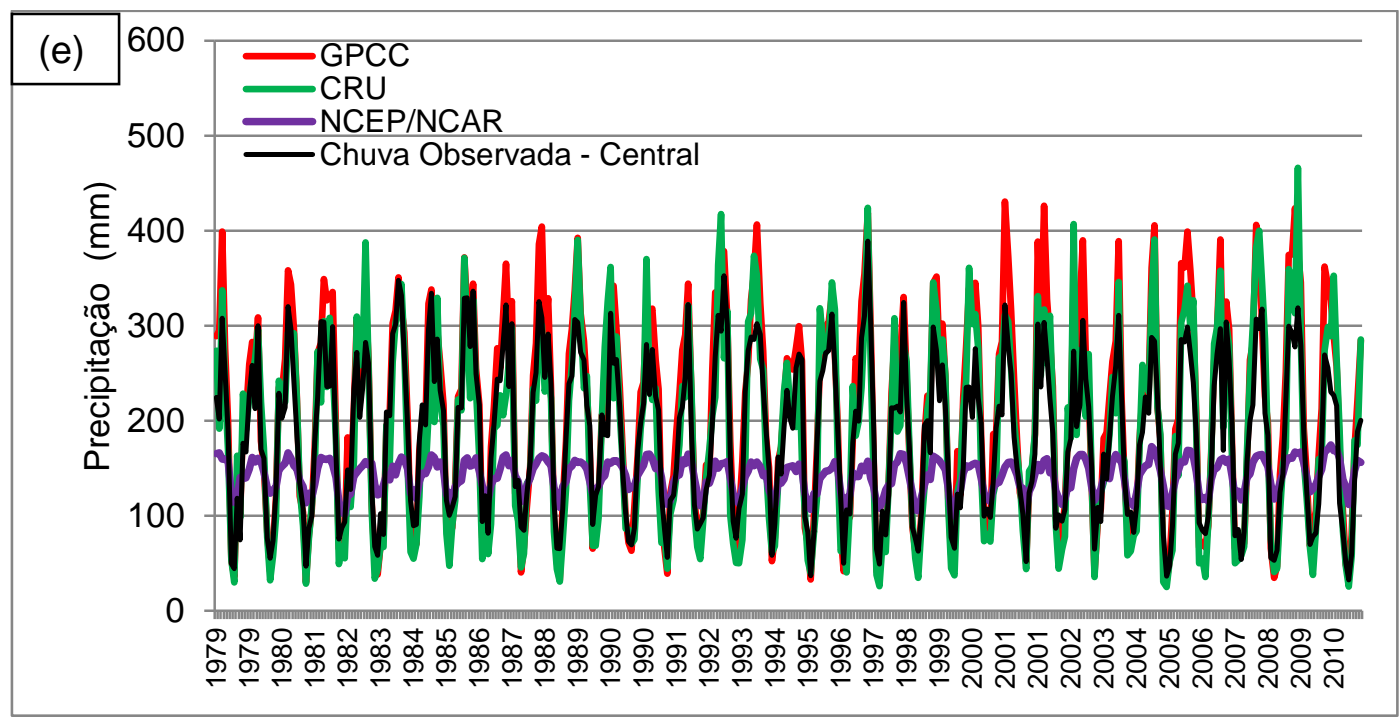

Figura 4 - Precipitação mensal referente aos dados do GPCC (linha vermelha), CRU (linha verde), Reanálise I do NCEP/NCAR (linha roxa) e ANA (linha preta), para as subregiões (a) Norte, (b) Amazonas-Foz, (c) Oeste, (d) Sul e (e) Central, entre 1979 e 2010.

Assim, enfatiza-se que os dados de precipitação do conjunto de dados do Reanálise I do NCEP/NCAR apresentam um comportamento bem diverso daquele apresentado pelos demais conjuntos de dados. Enquanto os dados de precipitação do CRU e GPCC apresentam sazonalidades semelhantes entre si e em relação aos dados observados da ANA, os dados de precipitação da Reanálise I do NCEP/NCAR apresentam uma amplitude sazonal bem menor. Atribui-se esta diferença ao fato de que os dados do NCEP/NCAR constituem resultados de modelo numérico que não satisfazem adequadamente o balanço de umidade (TRENBERTH et al., 2011; FASULLO, 2012), enquanto que os demais conjuntos são reconstruídos a partir de dados observados de precipitação. A variabilidade espúria dos dados de precipitação da Reanálise I do NCEP/NCAR também foi observada por Trenberth et al. (2005), para dados globais.

Observa-se também que os valores máximos de chuva do CRU são, em geral, levemente superestimados nas sub-regiões Norte e Amazonas-Foz em relação aos dados da ANA. O mesmo ocorre nas sub-regiões Sul e Central com os dados do GPCC.

Os dados do GPCC acompanham melhor a variabilidade da precipitação mensal dos dados da ANA, o que é mais evidente nas sub-regiões Norte e Central (Figura 4 (a) e (e), respectivamente).

\section{CONCLUSÕES}

A análise dos resultados permite verificar que os dados reconstruídos (GPCC, CRU e Reanálise I do NCEP/NCAR) representam a variabilidade temporal da chuva mensal nas cinco sub-regiões homogêneas da Amazônia brasileira definidas na presente pesquisa, em relação aos dados observados da ANA. Os 
dados de precipitação mensal do GPCC e os do CRU são os que mais se assemelham aos dados da ANA. A precipitação mensal da reanálise I do NCEP/NCAR apresenta as maiores diferenças em relação aos demais conjuntos de dados considerados. Apesar de os dados de precipitação do NCEP/NCAR apresentarem valor médio anual relativamente próximo dos dados médios dos demais conjuntos de dados para todas as sub-regiões consideradas, sua amplitude sazonal é bem inferior à representada nos demais conjuntos. $E$, embora a amplitude sazonal dos dados de precipitação da Reanálise I seja menor, a variabilidade mensal para o período de 1979 a 2010 é razoável, apresentando valores de correlação com os dados da ANA que variam entre 0,637 e 0,890 para todas as sub-regiões consideradas, o que se deve unicamente à representação do ciclo anual pelos dados da reanálise I do NCEP/NCAR.

Os resultados mostram que é razoável considerar os conjuntos de dados de precipitação reconstruída GPCC e CRU em análises dos aspectos climáticos para as cinco sub-regiões amazônicas, em especial, para a sub-região Sul, que apresentou os maiores valores de correlação linear com os dados observados da ANA. Ressalta-se que qualquer conjunto de dados, tanto observados quanto reconstruídos, ou provenientes de reanálises, podem apresentar problemas de consistência, devido a falhas na obtenção ou registro, às técnicas estatísticas empregadas para o preenchimento de falhas e interpolação, etc.. Assim, recomenda-se definir o conjunto de dados mais adequado ao estudo proposto através de análises estatísticas de comparação entre dados. Reforça-se também a praticidade da utilização de dados interpolados para grade regular, pois, a partir deles, é possível analisar áreas que dispõem de poucos postos de coleta, séries muito curtas ou séries com muitas falhas, o que não exclui a consideração de dados originais observados.

\section{AGRADECIMENTOS}

Os autores agradecem ao CNPq pela concessão de bolsa de Doutorado Processo n. 161279/2011-2.

\section{REFERÊNCIAS}

ADLER, R.F.; KIDD, C.; PETTY, G.; MORISSEY, M.; GOODMAN, M. Intercomparison of Global precipitation products: the third precipitation intercomparison project (PIP-3). Bulletin of the American Meteorological Society. Vol. 82, n. 7, p. 1377-1396, 2001.

BROHAN, P.; KENNEDY, J.J.; HARRIS, I.; TETT, S.F.B.; JONES, P.D. Uncertainty estimates in regional and global observed temperature changes: a new dataset from 1850. J. Geophysical Research, 111, 2006, p. D12106, DOI:10.1029/2005JD006548.

CARVALHO, L.M.V.; JONES, C.; POSADAS, A.N.D.; QUIROZ, R.; BOOKHAGEN, B.; LIEBMANN, B. Precipitation Characteristics of the South American Monsoon System derived from multiple datasets. Journal of Climate, 25, p. 4600-4620, 2012. DOI: $10.1175 / J C L I-D-11-00335.1$ 
DEE, D., FASULLO, J., SHEA, D., WALSH, J. The climate data guide: atmospheric reanalysis: overview \& comparison tables. Disponível em <https://climatedataguide.ucar.edu/climate-data/atmospheric-reanalysisoverview-comparison-tables>. Acesso em 09 ago. 2016.

FASULLO, J. A mechanism for land-ocean contrasts in global monsoon trends in a warming climate. Climate Dynamics. Vol. 39, p. 1137-1147, 2012.

FUNK, C. et al. The Climate hazards infrared precipitation with stations - a new environmental record for monitoring extremes. Nature: Scientific Data. Vol. 2, 2015. DOI: $10.1038 /$ sdata.2015.66.

GULIZIA, C.; CAMILLONI, I. A spatio-temporal comparative study of the representation of precipitation over South America derived by three gridded data sets. International Journal of Climatology (short communication), published online in Wiley Online Library, 2015. DOI: 10.1002/joc.4416.

HARADA et al. The JRA-55 Reanalysis: Representation of atmospheric circulation and climate variability. Journal of the Meteorological Society of Japan. Vol. 94, n. 3, p. 269-302, 2016. DOI:10.2151/jmsj.2016-015

HOLTON, J. R. An introduction to Dynamic Meteorology. Londres: Academic Press, 1992.

HUFFMAN, G. F. et al. The TRMM Multi-satellite Precipitation Analysis: quasiglobal, multi-year, combined-sensor precipitation estimates at fine scale. Journal of Hydrometeorology. Vol. 8, n. 1, p. 38-55, 2007.

KALNAY, E. et al. The NCEP/NCAR 40-year reanalysis project. Bulletin of American Meteorological Society, 77, 437-470, 1996.

LEIVAS, J. F. et al. Avaliação dos prognósticos de precipitação simulada pelo modelo BRAMS na Amazônia Ocidental na estação chuvosa. Acta Amazonica. Vol. 41, n. 3, p. 347-354, 2011.

LIMBERGER, L. Variabilidade da vazão de regiões homogêneas da bacia hidrográfica amazônica brasileira: teleconexões com a temperatura da superfície do mar (TSM) de 1976-2010. 2015. Tese (Doutorado). Faculdade de Filosofia, Letras e Ciências Humanas - USP. São Paulo, 483f, 2015.

LOS, S.O. Testing gridded land precipitation data and precipitation and runoff reanalyses (1982-2010) between $450 \mathrm{~S}$ and $450 \mathrm{~N}$ with normalised difference vegetation index data. Hydrology and Earth System Sciences. Vol. 19, p. 1713$1725,2015$.

MEEHL, G.A. et al. The WCRP CMIP3 multimodel dataset: a new era in climate change research. Bulletin of the American Meteorological Society. P.1383-1394, set. 2007.

RIENECKER, M.M.; et al. MERRA: NASA's Modern-ERA retrospective analysis for research and applications. Journal of Climate. Vo. 24, p. 3624-3648, 2011.

ROGERSON, Peter A. Métodos estatísticos para a Geografia: um guia para o estudante. $3^{a}$ ed. Porto Alegre: Bookman, 2012.

SAHA, S. et al. The NCEP Climate forecast system reanalysis. Bulletin of American Meteorological Society. Vol. 91, p. 1015-1057, 2010. 
SCHNEIDER, U.; et al. GPCC Full Data Reanalysis Version 6.0 at $0.5^{\circ}$ : Monthly Land-Surface Precipitation from Rain-Gauges built on GTS-based and Historic Data. 2011. DOI: 10.5676/DWD_GPCC/FD_M_V6_050.

SIMMONS, A.; UPPALA, S.; DEE, D.; KOBAYASHI, S. ERA-Interim: New ECMWF reanalysis products from 1989 onwards. ECMWF Newsletters. N. 110, p. 25-35, 2007.

Disponível

em

<http://www.ecmwf.int/sites/default/files/elibrary/2006/14615-newsletterno110-winter-200607.pdf>. Acesso em 09 ago. 2016.

TRENBERTH, K.E.; GUILLEMOT, C.J. Evaluation of the atmospheric moisture and hydrological cycle in the NCEP/NCAR reanalyses. Climate Dynamics. Vol. 14, n. 3, p. 213-231, mar. 1998.

TRENBERTH, K.E.; FASULLO, J.; SMITH, L. Trends and variability in columnintegrated atmospheric water vapor. Climate Dynamics. Vol. 24, p. 741-758, 2005.

TRENBERTH, K.E.; FASULLO, J.; MACKARO, J. Atmospheric moisture transports from ocean to land and global energy flows in reanalysis. Journal of Climate. Vol. 24, p. 4907-4924, 2011.

VAREJÃO-SILVA, M.A. Meteorologia e Climatologia. 2a. ed. digital. Disponível em<http://www.icat.ufal.br/laboratorio/clima/data/uploads/pdf/METEOROLOGIA _E_CLIMATOLOGIA_VD2_Mar_2006.pdf,>. Acesso em 21 mar. 2016.

YIN, X.; GRUBER, A.; ARKIN, P. Comparison of the GPCP and CMAP Merged gauge-satellite monthly precipitation products for the period 1979-2001. Journal of hydrometeorology. Vol. 5, p. 1207-1222, 2004.

ZVERYAEV, I.; CHU, P-S. Recent climate changes in precipitable water in global tropics as revealed in National Center for Environmental Prediction/National Center for Atmospheric Research reanalysis. Journal of Geophysics Research. Vol. 108, n. D10, p. ACL 6-1 - 6-12, 2003. DOI: 10.1029/2002JD002476. 